\title{
Exponential stability of travelling waves for a general reaction-diffusion equation with spatio-temporal delays
}

\author{
Rui Yan ${ }^{\mathrm{a}}$, Guirong Liu ${ }^{\mathrm{b}, *}$ \\ a School of Applied Mathematics, Shanxi University of Finance and Economics, Taiyuan 030006, China \\ b School of Mathematical Sciences, Shanxi University, Taiyuan 030006, China
}

*Corresponding author, e-mail: lgr5791@sxu.edu.cn

Received 10 Jun 2018

Accepted 31 Dec 2018

\begin{abstract}
This paper is concerned with the exponential stability of travelling waves for a general reaction-diffusion equation with spatio-temporal delays. Here the travelling waves may be monotone or non-monotone. More precisely, by means of the weighted-energy method and the nonlinear Halanay inequality, we can prove that the travelling waves of this equation are exponentially stable, when the initial perturbation around the wave is small in a weighted norm. In the end, we apply our results to some models.
\end{abstract}

KEYWORDS: delayed reaction-diffusion equation, weighted energy method, Halanay inequality

MSC2010: 35K57 35B35 35R10 92D25

\section{INTRODUCTION}

In this paper, we will study a general reactiondiffusion equation with spatio-temporal delays

$$
\begin{aligned}
& u_{t}(t, x)=d u_{x x}(t, x) \\
& +F\left(u(t, x), \int_{-\infty}^{\infty} J(x-y) f(u(t-r, y)) d y\right),
\end{aligned}
$$

$t \geqslant 0, x \in \mathbb{R}$, combined with the initial datum

$$
u(s, x)=u_{0}(s, x), \quad s \in[-r, 0], x \in \mathbb{R},
$$

where $f$ may not be monotone or quasi-monotone, $d>0$ and $r \geqslant 0$. Firstly, we give following assumptions for (1).

$\left(A_{1}\right) J(y) \geqslant 0, J(y)=J(-y), \int_{-\infty}^{\infty} J(y) \mathrm{d} y=1$, and $\int_{-\infty}^{\infty} J(y) \mathrm{e}^{\lambda y} \mathrm{~d} y<\infty$ for $\lambda>0$.

$\left(A_{2}\right)$ For $K>0, f \in C^{2}([0, K],[0, f(K)]), f(0)=0$, $f^{\prime}(0)>0,0<f(x) \leqslant f(K)$ for $x \in(0, K]$, and $|f(x)-f(y)| \leqslant f^{\prime}(0)|x-y|$ for $x, y \in[0, K]$.

$\left(A_{3}\right) F \in C^{2}([0, K] \times[0, f(K)], \mathbb{R}), \quad F(0,0)=0$, $F(K, f(K))=0, F(x, f(x))>0, \partial_{1} F(x, y) \leqslant 0$, $\partial_{2} F(x, y)>0, \quad F(x, y) \leqslant \partial_{1} F(0,0) x+$ $\partial_{2} F(0,0) y$, and $\partial_{i} F(x, y) \leqslant \partial_{i} F(0,0)$ for $(x, y) \in[0, K] \times[0, f(K)]$ when $i=1,2$, and $\partial_{1} F(x, y)=\frac{\partial F}{\partial x}(x, y), \partial_{2} F(x, y)=\frac{\partial F}{\partial y}(x, y)$.

$\left(A_{4}\right)$ There exist $0<\theta<K$ and $0<\eta<K$ such that $f$ is increasing on $[0, \theta]$ and $F(x, y)=0$ has a solution $x \in(0, \theta)$ for each $y \in(0, \eta)$.
$\left(A_{5}\right) \partial_{2} F(K, f(K)) f^{\prime}(K)+\partial_{1} F(K, f(K)) \leqslant 0$ for any $r>0$, or $\partial_{2} F(K, f(K)) f^{\prime}(K)+\partial_{1} F(K, f(K))>0$ for $0<r<\bar{r}$, when

$$
\begin{gathered}
\bar{r}=\frac{\pi-\arctan \left[-\left(\partial_{1} F(K, f(K))\right)^{-1} M\right]}{M}, \\
M=\sqrt{\left(\partial_{2} F(K, f(K)) f^{\prime}(K)\right)^{2}-\left(\partial_{1} F(K, f(K))\right)^{2}} .
\end{gathered}
$$

It is well known that reaction-diffusion equations with delays are often used to demonstrate practical phenomena. And travelling wave solutions of these equations have been studied universally because of their important applications. Throughout this paper, a travelling wave solution of (1) always refers to a pair $(\phi, c)$, where $\phi=\phi(\xi), \xi=x+c t$, is a function on $\mathbb{R}$ satisfying

$$
\begin{aligned}
& c \phi^{\prime}(\xi)=d \phi^{\prime \prime}(\xi) \\
& \quad+F\left(\phi(\xi), \int_{-\infty}^{\infty} J(y) f(\phi(\xi-y-c r)) d y\right) .
\end{aligned}
$$

Furthermore, if $\phi$ is monotone and bounded, we call it a travelling wavefront.

For reaction-diffusion equations, monotonicity and quasi-monotonicity of reaction terms are common hypotheses to ensure the existence of travelling wave solutions. Without these hypotheses, many authors have studied the existence of travelling wave solutions. For example, $\mathrm{Ma}^{1}$ established 
the existence of travelling wave solutions for a class of delayed nonlocal equations without quasimonotonicity by constructing two associated auxiliary functions and applying Schauder's fixed point theorem. Similar results can be obtained ${ }^{2,3}$, i.e., they proved the existence of non-monotone travelling wave solutions. Wang ${ }^{4}$ proved the existence of travelling wave solutions for (1) by using upperlower solutions for associated integral equations and Schauder's fixed point $\mathrm{t}$ heorem. Then for a class of non-monotone reaction-diffusion equation with spatiotemporal delays, $\mathrm{Xu}$ et $\mathrm{al}^{5}$ obtained the existence and uniqueness of travelling wave solutions by presenting a new method to construct a closed and convex set and using Schauder's fixed point theorem.

Besides the existence of travelling wave solutions of delayed reaction-diffusion equations, the stability of travelling waves has also attracted great attention. Let us provide some background on this topic in the literature. The first conclusion on the linearized stability of travelling wave solutions was established by Schaaf ${ }^{6}$ via the spectrum analysis method. Mei et $\mathrm{al}^{7}$ proved the nonlinear stability of the travelling wavefronts for the local Nicholson's blowflies equation with a discrete delay by using a technical weighted-energy method. Subsequently, many researchers used this method to obtain the stability of travelling wavefronts for a variety of monostable reaction-diffusion equations with delays ${ }^{8,9}$ and further improved the former results to obtain global stability of travelling wavefronts by using both the comparison principle and the technical weighted-energy method ${ }^{10,11}$.

Since the monotonicity of both the equations and the travelling wave solutions are necessary for these results of stability, therefore we cannot use the above methods to establish the stability of non-monotone travelling wave solutions. Wu et $\mathrm{al}^{12}$ firstly s tudied the a symptotic stability of the non-monotone travelling wave solutions of delayed reaction-diffusion equations with crossing-monostability by adopting two ideal weight functions. Subsequently, by using the technical weighted-energy method and the nonlinear Halanay's inequality, Lin et al ${ }^{13}$ obtained exponential decay estimate of all non-critical travelling wave solutions including the oscillating waves under some assumptions for a time-delayed reaction-diffusion equation. Since there is no result about stability of travelling wave solutions for (1), the main purpose of this study is the stability of the travelling wave solutions for (1) with non-monotone reaction terms.

\section{PRELIMINARIES AND MAIN RESULTS}

In this paper, we assume that $C>0$ represents a generic constant and $C_{i}>0$ denotes a particular constant. We let $I$ denote an interval, typically $I=\mathbb{R} . L^{2}(I)$ is the space of the square integrable functions on $I$ and $H^{k}(I)(k \geqslant 0)$ is the Sobolev space of $L^{2}$-functions $f(x)$ defined on $I$ satisfying $f^{(i)}(x) \in$ $L^{2}(I), i=1, \ldots, k . L_{\omega}^{2}(I)$ denotes the weighted $L^{2}$ space with a weight function $\omega, \omega: \mathbb{R} \rightarrow(0, \infty)$ is a locally integrable function, with norm defined by $\|f\|_{L_{\omega}^{2}(I)}=\left(\int_{I} \omega(x) f^{2}(x) \mathrm{d} x\right)^{1 / 2} . \quad H_{\omega}^{k}(I)$ is the weighted Sobolev space with the norm $\|f\|_{H_{\omega}^{k}(I)}=$ $\left(\sum_{i=0}^{k} \int_{I} \omega(x)\left|f^{(i)}(x)\right|^{2} \mathrm{~d} x\right)^{1 / 2}$. Furthermore, we let $T>0$ be a constant, $\mathscr{B}$ be a Banach space, and denote by $C([0, T] ; \mathscr{B})$ the space of the $\mathscr{B}$-valued continuous functions on $[0, T]$. The spaces of the $\mathscr{B}$-valued functions on $[0, \infty)$ can be defined similarly. Furthermore, the space $\mathscr{C}_{\text {unif }}[-r, T], 0<T \leqslant$ $\infty$, is defined by

$$
\mathscr{C}_{\text {unif }}[-r, T]:=\{v(t, x) \in C([-r, T] \times \mathbb{R})\}
$$

such that $\lim _{x \rightarrow \infty} \mathrm{e}^{\mu_{2} t} v(t, x)$ exists uniformly for $t \in$ $[-r, T]$ and $\mu_{2}>0$, a constant to be defined later, and

$$
\lim _{x \rightarrow \infty} v_{x}(t, x)=0, \lim _{x \rightarrow \infty} v_{x x}(t, x)=0
$$

uniformly with respect to $t \in[-r, T]$. The nonlinear Halanay inequality is given as follows.

Lemma 1 (Ref. 13) Let $z(t)$ be the solution of the following linear delay differential equation

$$
\begin{aligned}
z^{\prime}(t)+k_{1} z(t)-k_{2} z(t-r) & =\alpha f(z(t))+\beta g(z(t-r)), \\
z(s) & =z_{0}(s), \quad s \in[-r, 0],
\end{aligned}
$$

where $k_{1}, k_{2}, \alpha, \beta$ are any given numbers, and $f(z)$ and $g(z)$ satisfy

$$
\begin{gathered}
|f(z)| \leqslant C|z|^{m},|g(z)| \leqslant C|z|^{n}, m>1, n>1 . \\
\text { If }\left|k_{2}\right| \leqslant k_{1} \text { for } r>0 \text { or }\left|k_{2}\right|>k_{1} \text { for } 0<r<\bar{r} \\
\bar{r}=\frac{\pi-\arctan \left(k_{1}^{-1} \sqrt{\left|k_{2}\right|^{2}-k_{1}^{2}}\right)}{\sqrt{\left|k_{2}\right|^{2}-k_{1}^{2}}}
\end{gathered}
$$

then if $\left\|z_{0}\right\|_{L^{\infty}(-r, 0)} \ll 1$,

$$
|z(t)| \leqslant C\left\|z_{0}\right\|_{L^{\infty}(-r, 0)} \mathrm{e}^{-v t}, \quad t>0,
$$

for some $0<v=v\left(k_{1}, k_{2}, r\right)<k_{1}$. 


\section{Theorem 1 (existence of travelling waves)}

Assume that $\left(A_{1}\right)-\left(A_{4}\right)$ hold, there exist a minimal wave speed $c_{*}>0$ and a corresponding number $\lambda_{*}\left(c_{*}\right)$ satisfying $\mathscr{P}\left(\lambda_{*}, c_{*}\right)=0$ and $\frac{\partial}{\partial \lambda} \mathscr{P}\left(\lambda_{*}, c_{*}\right)=0$ where

$$
\begin{aligned}
\mathscr{P}(\lambda, c) & =d \lambda^{2}-c \lambda+\partial_{1} F(0,0) \\
& +\partial_{2} F(0,0) f^{\prime}(0) \int_{-\infty}^{\infty} J(y) \mathrm{e}^{-\lambda(y+c r)} \mathrm{d} y
\end{aligned}
$$

is the characteristic equation. For any $c \geqslant c_{*}$, then (1) admits a travelling wave solution $\phi(\xi), \xi=x+c t$, such that $0<\phi(\xi) \leqslant K, \phi(-\infty)=0$ and

$$
0<\liminf _{\xi \rightarrow \infty} \phi(\xi) \leqslant \limsup _{\xi \rightarrow \infty} \phi(\xi) \leqslant K .
$$

If $\partial_{1} F(x, y) \leqslant 0$, then $\lim _{\xi \rightarrow \infty} \phi(\xi)=K$. In addition, if $f$ is nondecreasing on $[0, K]$, then $\phi(\xi)$ is nondecreasing on $\mathbb{R}$. While for $0<c<c_{*}$, there has no travelling wave solution. Furthermore, when $c>c_{*}, \mathscr{P}(\lambda, c)=0$ has two distinct positive real roots $\lambda_{1}(c)$ and $\lambda_{2}(c)$ with $0<\lambda_{1}(c)<\lambda_{2}(c)$ such that

$$
\mathscr{P}(\lambda, c)<0 \text { for } \lambda_{1}<\lambda<\lambda_{2} \text {. }
$$

When $c=c_{*}$, it holds that

$$
\mathscr{P}\left(\lambda_{*}, c_{*}\right)=0 \text { for } \lambda_{1}=\lambda_{*}=\lambda_{2} .
$$

For any $c>c_{*}$, we define a weight function as

$$
\omega(x)=\mathrm{e}^{-2 \lambda_{*}\left(x-x_{0}\right)} \quad \text { with } x_{0} \gg 1,
$$

where $\lambda_{*}>0$ is defined in Theorem 1 and $x_{0}$ will be defined later. It is obvious that $\omega(x) \rightarrow \infty$ as $x \rightarrow-\infty$ and $\omega(x) \rightarrow 0$ as $x \rightarrow \infty$. Then we state the main result of this paper as follows.

\section{Theorem 2 (stability of travelling waves)}

Assume that $\left(A_{1}\right)-\left(A_{5}\right)$ hold. For the given travelling wave $\phi(\xi)$ of (1) with the speed $c>\tilde{c}$, where $\tilde{c}$ satisfies

$$
\begin{gathered}
\tilde{c} \lambda_{*}-\partial_{2} F(0,0) f^{\prime}(0)\left(\int_{-\infty}^{\infty} J(y) \mathrm{e}^{-2 \lambda_{*}(y+\tilde{c} r)} \mathrm{d} y\right)^{1 / 2} \\
=2 d \lambda_{*}^{2}+\partial_{1} F(0,0),
\end{gathered}
$$

if $u_{0}(s, x)-\phi(x+c s)$ is in $C\left([-r, 0] ; C(\mathbb{R}) \cap H_{\omega}^{2}(\mathbb{R})\right) \cap$ $L^{2}\left([-r, 0] ; H_{\omega}^{2}(\mathbb{R})\right)$ and

$$
v_{0, \infty}:=\lim _{x \rightarrow \infty}\left[u_{0}(s, x)-\phi(x+c s)\right] \in C[-r, 0]
$$

exists uniformly with $s \in[-r, 0]$, then there exist constants $\delta_{0}>0$ and $0<\mu<\min \left\{\mu_{1}, \mu_{2}\right\}, \mu_{1}, \mu_{2}$ will be defined later, such that when

$$
\begin{aligned}
\max _{s \in[-r, 0]}\left\|\left(u_{0}-\phi\right)(s)\right\|_{C}^{2}+\left\|\left(u_{0}-\phi\right)(0)\right\|_{H_{\omega}^{2}}^{2} & \\
& +\int_{-r}^{0}\left\|\left(u_{0}-\phi\right)(s)\right\|_{H_{\omega}^{2}}^{2} \mathrm{~d} s \leqslant \delta_{0}^{2},
\end{aligned}
$$

the unique solution $u(t, x)$ of (1) and (2) exists globally and $u(t, x)-\phi(x+c t)$ is in $C([-r, \infty) ; C(\mathbb{R}) \cap$ $\left.H_{\omega}^{2}(\mathbb{R})\right) \cap L^{2}\left([-r, \infty) ; H_{\omega}^{2}(\mathbb{R})\right) \cap \mathscr{C}_{\text {unif }}[-r, \infty)$ with

$$
\sup _{x \in \mathbb{R}}|u(t, x)-\phi(x+c t)| \leqslant \tilde{C} \mathrm{e}^{-\mu t}, \quad t \geqslant 0,
$$

where $\tilde{C}=\sqrt{C_{18}} \delta_{0}, C_{18}$ will be determined later.

Remark 1 By Hölder inequality,

$$
\int_{-\infty}^{\infty} J(y) \mathrm{e}^{-\lambda_{*} y} \mathrm{~d} y \leqslant\left(\int_{-\infty}^{\infty} J(y) \mathrm{e}^{-2 \lambda_{*} y} \mathrm{~d} y\right)^{1 / 2} .
$$

Then

$$
\begin{array}{r}
c_{*} \lambda_{*}-\partial_{2} F(0,0) f^{\prime}(0)\left(\int_{-\infty}^{\infty} J(y) \mathrm{e}^{-2 \lambda_{*}\left(y+c_{*} r\right)} \mathrm{d} y\right)^{1 / 2} \\
-2 d \lambda_{*}^{2}-\partial_{1} F(0,0) \leqslant 0,
\end{array}
$$

and we obtain that $\tilde{c} \geqslant c_{*}$.

\section{A PRIORI ESTIMATE}

For any $c>c_{*}$, let $\phi(\xi)$ be a travelling wave solution of (1) and $v(t, \xi)=u(t, x)-\phi(x+c t), v_{0}(s, \xi)=$ $u_{0}(s, x)-\phi(x+c s)$, where $\xi=x+c t$. Then it can be verified that $v(t, \xi)$ defined above satisfies

$$
\begin{aligned}
v_{t}(t, \xi) & +c v_{\xi}(t, \xi)-d v_{\xi \xi}(t, \xi)-\partial_{1} F(\xi) v(t, \xi) \\
= & \partial_{2} F(\xi) \int_{-\infty}^{\infty} J(y)[f(v+\phi)-f(\phi)] \mathrm{d} y+Q,
\end{aligned}
$$

with $v(s, \xi)=v_{0}(s, \xi),(s, \xi) \in[-r, 0] \times \mathbb{R}$, where $F(\xi)=F\left(\phi(\xi), \int_{-\infty}^{\infty} J(y) f(\phi(\xi-y-c r)) d y\right)$,

$$
\begin{aligned}
Q= & F\left(v(t, \xi)+\phi(\xi), \int_{-\infty}^{\infty} J(y) f(v+\phi) \mathrm{d} y\right) \\
& -F(\xi)-\partial_{1} F(\xi) v(t, \xi) \\
& -\partial_{2} F(\xi) \int_{-\infty}^{\infty} J(y)[f(v+\phi)-f(\phi)] \mathrm{d} y .
\end{aligned}
$$


For any $\tau \geqslant 0$ and $T \geqslant 0$, define a space

$$
\begin{aligned}
& X(\tau-r, T+\tau)=C\left([\tau-r, T+\tau] ; H_{\omega}^{2}(\mathbb{R})\right) \cap \\
& L^{2}\left([\tau-r, T+\tau] ; H_{\omega}^{2}(\mathbb{R})\right) \cap \mathscr{C}_{\text {unif }}[\tau-r, T+\tau]
\end{aligned}
$$

with the norm

$$
M_{\tau}(T)^{2}=\sup _{t \in[\tau-r, T+\tau]}\left(\|u(t)\|_{C}^{2}+\|u(t)\|_{H_{\omega}^{2}}^{2}\right),
$$

and denote $u(t)=u(t, \cdot)$ and $M(T)=M_{0}(T)$. The estimate of $v(t, \xi)$ in the space $H_{\omega}^{2}(\mathbb{R})$ is the following.

Lemma 2 If $v(t, \xi) \in X(-r, T)$, then

$$
\begin{array}{r}
\|v(t)\|_{L_{\omega}^{2}}^{2} \\
+\int_{0}^{t} \int_{-\infty}^{\infty} \mathrm{e}^{-2 \mu(t-s)}\left[B_{\eta, \mu, \omega}(\xi)-C_{1} M(T)\right] \omega v^{2} \mathrm{~d} \xi \mathrm{d} s \\
\quad \leqslant C_{3} \mathrm{e}^{-2 \mu t}\left(\left\|v_{0}(0)\right\|_{L_{\omega}^{2}}^{2}+\int_{-r}^{0}\left\|v_{0}(s)\right\|_{L_{\omega}^{2}}^{2} \mathrm{~d} s\right),
\end{array}
$$

where

$$
\begin{aligned}
B_{\eta, \mu, \omega}(\xi)= & A_{\eta, \omega}(\xi)-2 \mu-\frac{f^{\prime}(0)}{\eta} \partial_{2} F(0,0) \\
& \times\left(\mathrm{e}^{2 \mu r}-1\right) \int_{-\infty}^{\infty} J(y) \mathrm{e}^{-2 \lambda_{*}(y+c r)} \mathrm{d} y,
\end{aligned}
$$

$$
\begin{aligned}
A_{\eta, \omega}(\xi)= & -c \frac{\omega^{\prime}}{\omega}-d\left(\frac{\omega^{\prime}}{\omega}\right)^{2} \\
& -2 \partial_{1} F(\xi)-\eta f^{\prime}(0) \partial_{2} F(0,0) \\
- & \frac{1}{\eta} f^{\prime}(0) \partial_{2} F(0,0) \int_{-\infty}^{\infty} J(y) \frac{\omega(\xi+y+c r)}{\omega(\xi)} \mathrm{d} y,
\end{aligned}
$$

and $\mu, \eta, C_{i}, i=1,3$ are positive numbers to be defined later.

Proof: Multiplying (4) by $\omega(\xi) v(t, \xi) \mathrm{e}^{2 \mu t}$, we have

$$
\begin{aligned}
&\left(\frac{1}{2} \mathrm{e}^{2 \mu t} \omega v^{2}\right)_{t}+\mathrm{e}^{2 \mu t}\left(\frac{1}{2} c \omega v^{2}-d \omega v v_{\xi}\right)_{\xi} \\
&+\mathrm{e}^{2 \mu t} d \omega v_{\xi}^{2}+\mathrm{e}^{2 \mu t} d \omega^{\prime} v v_{\xi} \\
&+\left(-\frac{c}{2} \frac{\omega^{\prime}}{\omega}-\mu\right) \mathrm{e}^{2 \mu t} \omega v^{2}-\partial_{1} F(\xi) \mathrm{e}^{2 \mu t} \omega v^{2} \\
&=\mathrm{e}^{2 \mu t} \omega v \partial_{2} F(\xi) \int_{-\infty}^{\infty} J(y)[f(\phi+v)-f(\phi)] \mathrm{d} y \\
&+\mathrm{e}^{2 \mu t} \omega v Q .
\end{aligned}
$$

Further,

$$
\left|\mathrm{e}^{2 \mu t} d \omega^{\prime} v v_{\xi}\right| \leqslant \frac{1}{2} d \mathrm{e}^{2 \mu t} \omega v_{\xi}^{2}+\frac{d}{2}\left(\frac{\omega^{\prime}}{\omega}\right)^{2} \mathrm{e}^{2 \mu t} \omega v^{2} .
$$

Thus (7) is changed into

$$
\begin{gathered}
\left(\frac{1}{2} \mathrm{e}^{2 \mu t} \omega \nu^{2}\right)_{t}+\frac{1}{2} d \mathrm{e}^{2 \mu t} \omega v_{\xi}^{2} \\
+\mathrm{e}^{2 \mu t}\left(\frac{1}{2} c \omega v^{2}-d \omega v v_{\xi}\right)_{\xi} \\
+\left(-\frac{c}{2} \frac{\omega^{\prime}}{\omega}-\mu-\frac{d}{2}\left(\frac{\omega^{\prime}}{\omega}\right)^{2}-\partial_{1} F(\xi)\right) \mathrm{e}^{2 \mu t} \omega v^{2} \\
\leqslant \mathrm{e}^{2 \mu t} \omega v \partial_{2} F(\xi) \int_{-\infty}^{\infty} J(y)[f(\phi+v)-f(\phi)] \mathrm{d} y \\
+\mathrm{e}^{2 \mu t} \omega v Q .
\end{gathered}
$$

Integrating (8) over $\mathbb{R} \times[0, t]$ with respect to $\xi$ and $t$ and note that $\left.(\sqrt{\omega} v)\right|_{\xi= \pm \infty}=\left.\left(\sqrt{\omega} v_{\xi}\right)\right|_{\xi= \pm \infty}=0$, we obtain

$$
\begin{aligned}
& \mathrm{e}^{2 \mu t}\|v(t)\|_{L_{\omega}^{2}(\mathbb{R})}^{2}+d \int_{0}^{t} \mathrm{e}^{2 \mu s}\left\|v_{\xi}(s)\right\|_{L_{\omega}^{2}(\mathbb{R})}^{2} \mathrm{~d} s \\
& +\int_{0}^{t} \int_{-\infty}^{\infty}\left(-c \frac{\omega^{\prime}}{\omega}-2 \mu-d\left(\frac{\omega^{\prime}}{\omega}\right)^{2}-2 \partial_{1} F(\xi)\right) \\
& \times \mathrm{e}^{2 \mu s} \omega(\xi) v^{2}(s, \xi) \mathrm{d} \xi \mathrm{d} s \\
& \leqslant\left\|v_{0}(0)\right\|_{L_{\omega}^{2}(\mathbb{R})}^{2}+2 \int_{0}^{t} \int_{-\infty}^{\infty} \mathrm{e}^{2 \mu s} \omega(\xi) v(s, \xi) Q \mathrm{~d} \xi \mathrm{d} s \\
& +2 \int_{0}^{t} \int_{-\infty}^{\infty} \mathrm{e}^{2 \mu s} \omega(\xi) v(s, \xi) \partial_{2} F(\xi) \\
& \quad \times \int_{-\infty}^{\infty} J(y)(f(v+\phi)-f(\phi)) \mathrm{d} y \mathrm{~d} \xi \mathrm{d} s .
\end{aligned}
$$

Note that

$$
\begin{aligned}
2|v(s, \xi)| \partial_{2} F(\xi) \int_{-\infty}^{\infty} J(y)|f(v+\phi)-f(\phi)| \mathrm{d} y \\
\quad \leqslant \partial_{2} F(0,0) f^{\prime}(0) \int_{-\infty}^{\infty} J(y) \\
\times\left(\eta v^{2}(s, \xi)+\frac{1}{\eta} v^{2}(s-r, \xi-y-c r)\right) \mathrm{d} y
\end{aligned}
$$

where $\eta>0$ will be specified later and

$$
\begin{aligned}
& \frac{1}{\eta} \int_{0}^{t} \int_{-\infty}^{\infty} \mathrm{e}^{2 \mu s} \omega(\xi) \\
& \quad \times \int_{-\infty}^{\infty} J(y) v^{2}(s-r, \xi-y-c r) \mathrm{d} y \mathrm{~d} \xi \mathrm{d} s \\
& \leqslant \frac{1}{\eta} \mathrm{e}^{2 \mu r} \int_{0}^{t} \int_{-\infty}^{\infty} \int_{-\infty}^{\infty} \mathrm{e}^{2 \mu s} \omega(\xi+y+c r) \\
& \quad \times J(y) v^{2}(s, \xi) \mathrm{d} y \mathrm{~d} \xi \mathrm{d} s \\
& +\frac{1}{\eta} \mathrm{e}^{2 \mu r} \int_{-r}^{0} \int_{-\infty}^{\infty} \int_{-\infty}^{\infty} \mathrm{e}^{2 \mu s} \omega(\xi+y+c r) \\
& \times J(y) v_{0}^{2}(s, \xi) \mathrm{d} y \mathrm{~d} \xi \mathrm{d} s .
\end{aligned}
$$


Hence we obtain

$$
\begin{aligned}
& 2 \int_{0}^{t} \int_{-\infty}^{\infty} \mathrm{e}^{2 \mu s} \omega(\xi)|v(s, \xi)| \partial_{2} F(\xi) \\
& \quad \times \int_{-\infty}^{\infty} J(y)|f(v+\phi)-f(\phi)| \mathrm{d} y \mathrm{~d} \xi \mathrm{d} s \\
& \leqslant \eta \partial_{2} F(0,0) f^{\prime}(0) \int_{0}^{t} \int_{-\infty}^{\infty} \mathrm{e}^{2 \mu s} \omega(\xi) v^{2}(s, \xi) \mathrm{d} \xi \mathrm{d} s \\
& +\frac{1}{\eta} \partial_{2} F(0,0) f^{\prime}(0) \mathrm{e}^{2 \mu r} \\
& \times \int_{0}^{t} \int_{-\infty}^{\infty} \int_{-\infty}^{\infty} J(y) \mathrm{e}^{2 \mu s} \omega(\xi+y+c r) v^{2}(s, \xi) \mathrm{d} y \mathrm{~d} \xi \mathrm{d} s \\
& \times \int_{-r}^{0} \int_{-\infty}^{\infty} \int_{-\infty}^{\infty} J(y) \mathrm{e}^{2 \mu s} \omega(\xi+y+c r) v_{0}^{2}(s, \xi) \mathrm{d} y \mathrm{~d} \xi \mathrm{d} s .
\end{aligned}
$$

For the nonlinearity $Q$, by applying Taylor's formula to (5), it follows that

$$
\begin{aligned}
Q & =\frac{1}{2} \partial_{11} F(\bar{\phi}, \tilde{\phi}) v^{2} \\
& +\partial_{12} F(\bar{\phi}, \tilde{\phi})\left[\int_{-\infty}^{\infty} J(y)[f(v+\phi)-f(\phi)] \mathrm{d} y\right] v \\
& +\frac{1}{2} \partial_{22} F(\bar{\phi}, \tilde{\phi})\left[\int_{-\infty}^{\infty} J(y)[f(v+\phi)-f(\phi)] \mathrm{d} y\right]^{2},
\end{aligned}
$$

where $\bar{\phi}$ is between $\phi$ and $\phi+v$ and $\tilde{\phi}$ is between $\int_{-\infty}^{\infty} J(y) f(\phi(\xi-y-c r)) \mathrm{d} y$ and $\int_{-\infty}^{\infty} J(y) f(v+$ $\phi) \mathrm{d} y$. For the third term on the right-hand side of (11), using Hölder's inequality gives

$$
\begin{aligned}
& \left|\int_{-\infty}^{\infty} J(y)[f(v+\phi)-f(\phi)] \mathrm{d} y\right| \\
& \quad \leqslant \int_{-\infty}^{\infty} J(y) f^{\prime}(0)|v(s-r, \xi-y-c r)| \mathrm{d} y \\
& \quad \leqslant\left(\int_{-\infty}^{\infty} J(y)\left(f^{\prime}(0) v(s-r, \xi-y-c r)\right)^{2} \mathrm{~d} y\right)^{1 / 2} .
\end{aligned}
$$

Because of the definition of $M(T)$ and $A_{3}$, then

$$
\begin{aligned}
& \left|2 \int_{0}^{t} \int_{-\infty}^{\infty} \mathrm{e}^{2 \mu s} \omega(\xi) v(s, \xi) Q \mathrm{~d} \xi \mathrm{d} s\right| \\
& \leqslant C_{1} M(T) \int_{0}^{t} \int_{-\infty}^{\infty} \mathrm{e}^{2 \mu s} \omega(\xi) v^{2}(s, \xi) \mathrm{d} \xi \mathrm{d} s \\
& \quad+C_{2} \int_{-r}^{0} \int_{-\infty}^{\infty} \mathrm{e}^{2 \mu s} \omega(\xi) v_{0}^{2}(s, \xi) \mathrm{d} \xi \mathrm{d} s,
\end{aligned}
$$

where

$$
\begin{aligned}
& C_{1}=L\left(1+f^{\prime}(0)+2 f^{\prime}(0) \mathrm{e}^{2 \mu r} \int_{-\infty}^{\infty} J(y) \mathrm{e}^{-2 \lambda_{*} y} \mathrm{~d} y\right), \\
& C_{2}=2 L M(T) f^{\prime}(0) \mathrm{e}^{2 \mu r} \int_{-\infty}^{\infty} J(y) \mathrm{e}^{-2 \lambda_{*} y} \mathrm{~d} y
\end{aligned}
$$

and $L=\max \left\{\left|\partial_{i j} F(x, y)\right|\right\}$ for $(x, y) \in[0, K] \times$ $[0, f(K)], i, j=1,2$.

Substituting (10) and (12) into (9), we obtain

$$
\begin{aligned}
& \mathrm{e}^{2 \mu t}\|v(t)\|_{L_{\omega}^{2}}^{2}+d \int_{0}^{t} \mathrm{e}^{2 \mu s}\left\|v_{\xi}(s)\right\|_{L_{\omega}^{2}}^{2} \mathrm{~d} s \\
& +\int_{0}^{t} \int_{-\infty}^{\infty} \mathrm{e}^{2 \mu s}\left(B_{\eta, \mu, \omega}(\xi)-C_{1} M(T)\right) \omega v^{2} \mathrm{~d} \xi \mathrm{d} s \\
& \leqslant C_{3}\left(\left\|v_{0}(0)\right\|_{L_{\omega}^{2}}^{2}+\int_{-r}^{0}\left\|v_{0}(s)\right\|_{L_{\omega}^{2}}^{2} \mathrm{~d} s\right),
\end{aligned}
$$

where $B_{\eta, \mu, \omega}(\xi)$ is given in (6) and $C_{3}=\max \left\{1, C_{2}+\right.$ $\left.\left(f^{\prime}(0) / \eta\right) \partial_{2} F(0,0) \mathrm{e}^{2 \mu r} \int_{-\infty}^{\infty} J(y) \mathrm{e}^{-2 \lambda_{*} y} \mathrm{~d} y\right\}$.

Lemma 3 Let $\eta=\left(\int_{-\infty}^{\infty} J(y) \mathrm{e}^{-2 \lambda_{*}(y+c r)} \mathrm{d} y\right)^{1 / 2}$. Then for $c>\tilde{c}$, we have $A_{\eta, \omega}(\xi) \geqslant C_{4}>0$ for $\xi \in \mathbb{R}$.

Proof: Since $\omega(\xi)=\mathrm{e}^{-2 \lambda_{*}\left(\xi-x_{0}\right)}, \omega^{\prime}(\xi) / \omega(\xi)=$ $-2 \lambda_{*}, \omega(\xi+y+c r) / \omega(\xi)=\mathrm{e}^{-2 \lambda_{*}(y+c r)}$ and $c>\tilde{c}$,

$$
\begin{aligned}
A_{\eta, \omega} & (\xi) \\
\geqslant & 2 c \lambda_{*}-4 d \lambda_{*}^{2}-2 \partial_{1} F(0,0)-\eta \partial_{2} F(0,0) f^{\prime}(0) \\
& \quad-\frac{1}{\eta} \partial_{2} F(0,0) f^{\prime}(0) \int_{-\infty}^{\infty} J(y) \mathrm{e}^{-2 \lambda_{*}(y+c r)} \mathrm{d} y \\
= & : C_{4}>0 .
\end{aligned}
$$

Lemma 4 Let $v(t, \xi) \in X(-r, T)$ and $\mu_{1}>0$ be the unique root of the equation

$$
\begin{aligned}
C_{4}-2 \mu-\partial_{2} F(0,0) f^{\prime}(0)\left(\mathrm{e}^{2 \mu r}-1\right) & \\
& \times\left(\int_{-\infty}^{\infty} J(y) \mathrm{e}^{-2 \lambda_{*}(y+c r)} \mathrm{d} y\right)^{1 / 2}=0 .
\end{aligned}
$$

Then for $0<\mu<\mu_{1}$, we have

$$
\begin{aligned}
& \|v(t)\|_{L_{\omega}^{2}}^{2}+\int_{0}^{t} \mathrm{e}^{-2 \mu(t-s)}\|v(s)\|_{L_{\omega}^{2}}^{2} \mathrm{~d} s \\
& \leqslant C_{6} \mathrm{e}^{-2 \mu t}\left(\left\|v_{0}(0)\right\|_{L_{\omega}^{2}}^{2}+\int_{-r}^{0}\left\|v_{0}(s)\right\|_{L_{\omega}^{2}}^{2} \mathrm{~d} s\right),
\end{aligned}
$$

where $M(T) \ll 1$ and $C_{6}$ will be defined later. 
Proof: By Lemma 3 we have that for $0<\mu<\mu_{1}$,

$$
\begin{aligned}
B_{\eta, \mu, \omega}(\xi) \geqslant C_{4} & -2 \mu-\partial_{2} F(0,0) f^{\prime}(0)\left(\mathrm{e}^{2 \mu r}-1\right) \\
& \times\left(\int_{-\infty}^{\infty} J(y) \mathrm{e}^{-2 \lambda_{*}(y+c r)} \mathrm{d} y\right)^{1 / 2} \\
=: & C_{5}>0
\end{aligned}
$$

Substitute (15) into (13) and let $0<M(T)<C_{5} / C_{1}$ be small enough, then

$$
\begin{aligned}
& \mathrm{e}^{2 \mu t}\|v(t)\|_{L_{\omega}^{2}}^{2}+ \int_{0}^{t} \mathrm{e}^{2 \mu s}\left\|v_{\xi}(s)\right\|_{L_{\omega}^{2}}^{2} \mathrm{~d} s \\
&+\int_{0}^{t} \mathrm{e}^{2 \mu s}\|v(s)\|_{L_{\omega}^{2}}^{2} \mathrm{~d} s \\
& \leqslant C_{6}\left(\left\|v_{0}(0)\right\|_{L_{\omega}^{2}}^{2}+\int_{-r}^{0}\left\|v_{0}(s)\right\|_{L_{\omega}^{2}}^{2} \mathrm{~d} s\right),
\end{aligned}
$$

where $C_{6}:=C_{3} / \min \left\{1, d, C_{5}-C_{1} M(T)\right\}$.

Lemma 5 Let $v(t, \xi) \in X(-r, T)$. Then for $c>\tilde{c}$,

$$
\begin{aligned}
& \left\|v_{\xi}(t)\right\|_{L_{\omega}^{2}}^{2}+\int_{0}^{t} \mathrm{e}^{-2 \mu(t-s)}\left\|v_{\xi}(s)\right\|_{L_{\omega}^{2}}^{2} \mathrm{~d} s \\
& \leqslant C_{11} \mathrm{e}^{-2 \mu t}\left(\left\|v_{0}(0)\right\|_{H_{\omega}^{1}}^{2}+\int_{-r}^{0}\left\|v_{0}(s)\right\|_{H_{\omega}^{1}}^{2} \mathrm{~d} s\right) .
\end{aligned}
$$

Proof: By differentiating (4) with $\xi$ and multiplying it by $\mathrm{e}^{2 \mu t} \omega(\xi) \nu_{\xi}(t, \xi)$, we have

$$
\begin{gathered}
\left(\frac{1}{2} \mathrm{e}^{2 \mu t} \omega v_{\xi}^{2}\right)_{t}+\frac{d}{2} \mathrm{e}^{2 \mu t} \omega v_{\xi \xi}^{2} \\
+\mathrm{e}^{2 \mu t}\left(\frac{1}{2} c \omega v_{\xi}^{2}-d \omega v_{\xi} v_{\xi \xi}\right)_{\xi} \\
+\left(-\frac{d}{2}\left(\frac{\omega^{\prime}}{\omega}\right)^{2}-\frac{c}{2} \frac{\omega^{\prime}}{\omega}-\mu-\partial_{1} F(\xi)\right) \mathrm{e}^{2 \mu t} \omega v_{\xi}^{2} \\
\leqslant\left[\partial_{1} F(\xi)\right]_{\xi} \mathrm{e}^{2 \mu t} \omega v v_{\xi}+\mathrm{e}^{2 \mu t} \omega v_{\xi} \\
\times\left(\partial_{2} F(\xi) \int_{-\infty}^{\infty} J(y)[f(\phi+v)-f(\phi)] \mathrm{d} y+Q\right)_{\xi} .
\end{gathered}
$$

By integrating (18) with respect to $\xi$ and $t$ over $\mathbb{R} \times$
$[0, t]$, we obtain

$$
\begin{aligned}
& \mathrm{e}^{2 \mu t}\left\|v_{\xi}(t)\right\|_{L_{\omega}^{2}}^{2}+d \int_{0}^{t} \mathrm{e}^{2 \mu s}\left\|v_{\xi \xi}(s)\right\|_{L_{\omega}^{2}}^{2} \mathrm{~d} s \\
& +\int_{0}^{t} \int_{-\infty}^{\infty}\left\{-d\left(\frac{\omega^{\prime}}{\omega}\right)^{2}-c \frac{\omega^{\prime}}{\omega}-2 \mu-2 \partial_{1} F(\xi)\right\} \\
& \leqslant \int_{0}^{t} \int_{-\infty}^{\infty} 2\left\{\left[\mathrm{e}^{2 \mu s} \omega v_{\xi}^{2} \mathrm{~d} \xi \mathrm{d} s\right.\right. \\
& +\int_{0}^{t} \int_{-\infty}^{\infty} \int_{-\infty}^{\infty} J(y)[f(\phi+v)-f(\phi)]_{\xi} \mathrm{d} y \\
& \times 2 \partial_{2} F(\xi) \mathrm{e}^{2 \mu s} \omega v_{\xi} \mathrm{d} \xi \mathrm{d} s .
\end{aligned}
$$

Note that

$$
\begin{aligned}
& {\left[\partial_{1} F(\xi)\right]_{\xi} v} \\
& +\left(\partial_{2} F(\xi) \int_{-\infty}^{\infty} J(y)[f(\phi+v)-f(\phi)] \mathrm{d} y+Q\right)_{\xi} \\
& =\left\{\partial_{1} F\left(\bar{\varphi}, \int_{-\infty}^{\infty} J(y) f(\phi+v) \mathrm{d} y\right) v\right\}_{\xi}-\partial_{1} F(\xi) v_{\xi}^{2} \\
& +\left\{\partial_{2} F(\phi, \tilde{\varphi}) \int_{-\infty}^{\infty} J(y)[f(\phi+v)-f(\phi)] \mathrm{d} y\right\}_{\xi} \\
& +\partial_{2} F(\xi)\left(\int_{-\infty}^{\infty} J(y)[f(\phi+v)-f(\phi)] \mathrm{d} y\right)_{\xi} \\
& \quad-\partial_{2} F(\xi)\left(\int_{-\infty}^{\infty} J(y)[f(\phi+v)-f(\phi)] \mathrm{d} y\right)_{\xi}
\end{aligned}
$$

where $\bar{\varphi}=\phi+\left(1-\theta_{1}\right) v, \tilde{\varphi}=\theta_{2} \int_{-\infty}^{\infty} J(y) f(\phi) \mathrm{d} y+$ $\left(1-\theta_{2}\right) \int_{-\infty}^{\infty} J(y) f(\phi+v) \mathrm{d} y$ with $\theta_{1}, \theta_{2} \in[0,1]$. Hence (19) becomes

$$
\begin{aligned}
& \mathrm{e}^{2 \mu t}\left\|v_{\xi}(t)\right\|_{L_{\omega}^{2}}^{2}+d \int_{0}^{t} \mathrm{e}^{2 \mu s}\left\|v_{\xi \xi}(s)\right\|_{L_{\omega}^{2}}^{2} \mathrm{~d} s \\
& \quad+\int_{0}^{t} \int_{-\infty}^{\infty} B_{\eta, \mu, \omega}(\xi) \mathrm{e}^{2 \mu s} \omega v_{\xi}^{2} \mathrm{~d} \xi \mathrm{d} s \\
& \leqslant C_{7} \int_{0}^{t} \mathrm{e}^{2 \mu s}\|v(s)\|_{L_{\omega}^{2}}^{2} \mathrm{~d} s+C_{8} \int_{0}^{t} \mathrm{e}^{2 \mu s}\left\|v_{\xi}(s)\right\|_{L_{\omega}^{2}}^{2} \mathrm{~d} s \\
& +C_{9} \int_{-r}^{0}\left\|v_{0}(s)\right\|_{L_{\omega}^{2}}^{2} \mathrm{~d} s+C_{10} \int_{-r}^{0}\left\|v_{0 \xi}(s)\right\|_{L_{\omega}^{2}}^{2} \mathrm{~d} s .
\end{aligned}
$$

Combining with (16), it holds that

$$
\begin{aligned}
\mathrm{e}^{2 \mu t}\left\|v_{\xi}(t)\right\|_{L_{\omega}^{2}}^{2} & +\int_{0}^{t} \mathrm{e}^{2 \mu s}\left(\left\|v_{\xi \xi}(s)\right\|_{L_{\omega}^{2}}^{2}\left\|v_{\xi}(s)\right\|_{L_{\omega}^{2}}^{2}\right) \mathrm{d} s \\
& \leqslant C_{11}\left(\left\|v_{0}(0)\right\|_{H_{\omega}^{1}}^{2}+\int_{-r}^{0}\left\|v_{0}(s)\right\|_{H_{\omega}^{1}}^{2} \mathrm{~d} s\right),
\end{aligned}
$$


where $C_{11}=\frac{\max \left\{C_{6} C_{7}+C_{6} C_{8}+C_{9}, C_{10}\right\}}{\min \left\{1, d, C_{5}\right\}}$.

Similarly, we obtain the estimate of $v_{\xi \xi}(t, \xi)$.

Lemma 6 Let $v(t, \xi) \in X(-r, T)$. Then for $c>\tilde{c}$, there exists $C_{12}>0$ such that

$$
\begin{aligned}
& \left\|v_{\xi \xi}(t)\right\|_{L_{\omega}^{2}}^{2}+\int_{0}^{t} \mathrm{e}^{-2 \mu(t-s)}\left\|v_{\xi \xi}(s)\right\|_{L_{\omega}^{2}}^{2} \mathrm{~d} s \\
& \leqslant C_{12} \mathrm{e}^{-2 \mu t}\left(\left\|v_{0}(0)\right\|_{H_{\omega}^{2}}^{2}+\int_{-r}^{0}\left\|v_{0}(s)\right\|_{H_{\omega}^{2}}^{2} \mathrm{~d} s\right),
\end{aligned}
$$

on condition that $M(T) \ll 1$.

By combining with (14), (17) and (21), we obtain the lemma.

Lemma 7 Let $v(t, \xi) \in X(-r, T)$. Then for $c>\tilde{c}$,

$$
\begin{aligned}
& \|v(t)\|_{H_{\omega}^{2}}^{2}+\int_{0}^{t} \mathrm{e}^{-2 \mu(t-s)}\|v(s)\|_{H_{\omega}^{2}}^{2} \mathrm{~d} s \\
& \leqslant C_{13} \mathrm{e}^{-2 \mu t}\left(\left\|v_{0}(0)\right\|_{H_{\omega}^{2}}^{2}+\int_{-r}^{0}\left\|v_{0}(s)\right\|_{H_{\omega}^{2}}^{2} \mathrm{~d} s\right),
\end{aligned}
$$

when $M(T) \ll 1$ and $C_{13}=\max \left\{C_{6}, C_{11}, C_{12}\right\}$.

By applying the Sobolev embedding theorem $H^{2}(\mathbb{R}) \hookrightarrow C^{1}(\mathbb{R})$ and noting that $\omega(\xi) \geqslant 1$ for $\xi \in$ $\left(-\infty, x_{0}\right], x_{0}$ will be defined later, we obtain the following decay results.

Lemma 8 Let $v(t, \xi) \in X(-r, T)$. For $t \in[0, T]$,

$$
\begin{gathered}
\sup _{\xi \in\left(-\infty, x_{0}\right]}|v(t, \xi)| \leqslant C_{14}\|v(t)\|_{H^{2}} \leqslant C_{14}\|v(t)\|_{H_{\omega}^{2}} \\
\leqslant C_{15}\left(\left\|v_{0}(0)\right\|_{H_{\omega}^{2}}^{2}+\int_{-r}^{0}\left\|v_{0}(s)\right\|_{H_{\omega}^{2}}^{2} \mathrm{~d} s\right)^{1 / 2} \mathrm{e}^{-\mu t},
\end{gathered}
$$

where $C_{14}$ is the embedding constant and $C_{15}=$ $C_{14} \sqrt{C_{13}}$.

To prove the exponential decay of $v(t, \xi)$ for $\xi \in\left[x_{0}, \infty\right)$, we first show the result of $v(t, \xi)$ when $\xi=\infty$. For $v(t, \xi) \in X(-r, T), \lim _{\xi \rightarrow \infty} v(t, \xi)$ exists uniformly and $\lim _{\xi \rightarrow \infty} v_{\xi}(t, \xi)=\lim _{\xi \rightarrow \infty} v_{\xi \xi}(t, \xi)=0$ uniformly for $t \in[0, T]$. We define $z(t):=v(t, \infty)$, $z_{0}(s):=v_{0}(s, \infty)$ for $s \in[-r, 0]$. Furthermore, we verify that $v(t, \xi)$ satisfies

$$
\begin{aligned}
& v_{t}(t, \xi)+c v_{\xi}(t, \xi)-d v_{\xi \xi}(t, \xi)-\partial_{1} F(\xi) v(t, \xi) \\
& \quad=\partial_{2} F(\xi) \int_{-\infty}^{\infty} J(y) f^{\prime}(\phi) v \mathrm{~d} y+\tilde{Q}(v) \\
& v(s, \xi)=v_{0}(s, \xi),(s, \xi) \in[-r, 0] \times \mathbb{R}
\end{aligned}
$$

where

$$
\begin{gathered}
F(\xi)=F\left(\phi(\xi), \int_{-\infty}^{\infty} J(y) f(\phi(\xi-y-c r)) \mathrm{d} y\right), \\
\tilde{Q}(v)=F\left(v+\phi, \int_{-\infty}^{\infty} J(y) f(v+\phi) \mathrm{d} y\right)-F(\xi) \\
-\partial_{1} F(\xi) v(t, \xi)-\partial_{2} F(\xi) \int_{-\infty}^{\infty} J(y) f^{\prime}(\phi) v \mathrm{~d} y .
\end{gathered}
$$

By letting $\xi \rightarrow \infty$ to (24), we have

$$
\begin{aligned}
& z^{\prime}(t)-\partial_{1} F(K, f(K)) z(t) \\
& -\partial_{2} F(K, f(K)) f^{\prime}(K) z(t-r)=\tilde{Q}(z), \\
& z(s)=z_{0}(s), \quad s \in[-r, 0] .
\end{aligned}
$$

Lemma 9 If $\left(A_{5}\right)$ holds and $z(t)$ is the solution of (25), then there exists $C_{16}>0$ such that

$$
|z(t)| \leqslant C_{16} M(0) \mathrm{e}^{-\mu_{2} t}, \quad t>0,
$$

when $0<\mu_{2}<-\partial_{1} F(K, f(K))$ and $M(0) \ll 1$.

Proof: Let $l_{1}=-\partial_{1} F(K, f(K)) \geqslant 0$ and $l_{2}=$ $\partial_{2} F(K, f(K)) f^{\prime}(K)>0$. It follows from Lemma 1 that if $l_{2} \leqslant l_{1}$ with any $r>0$, or if $l_{2}>l_{1}$ with $0<r<\bar{r}$, then there exists $C_{16}>0$ such that

$$
|z(t)| \leqslant C_{16} M(0) \mathrm{e}^{-\mu_{2} t}, \quad t>0,
$$

with some constant $0<\mu_{2}=\mu_{2}\left(l_{1}, l_{2}, r\right)<l_{1}$, if $M(0) \ll 1$ and $\bar{r}$ is defined in (3).

Note that $\lim _{\xi \rightarrow \infty}\left|\mathrm{e}^{\mu_{2} t} v(t, \xi)-\mathrm{e}^{\mu_{2} t} v(t, \infty)\right|=0$ uniformly for $t \in[0, T]$. Then for $\epsilon=M(0)$, there exists $x_{0}=x_{0}(M(0)) \gg 1$ such that $\mid \mathrm{e}^{\mu_{2} t} v(t, \xi)-$ $\mathrm{e}^{\mu_{2} t} v(t, \infty) \mid<\epsilon$ if $\xi \geqslant x_{0}$. That is,

$$
\begin{aligned}
\left|\mathrm{e}^{\mu_{2} t}\right| v(t, \xi)\left|-\mathrm{e}^{\mu_{2} t}\right| v(t, \infty)|| & \\
& \leqslant\left|\mathrm{e}^{\mu_{2} t} v(t, \xi)-\mathrm{e}^{\mu_{2} t} v(t, \infty)\right|<\epsilon .
\end{aligned}
$$

Thus from Lemma 9,

$\mathrm{e}^{\mu_{2} t}|v(t, \xi)| \leqslant C_{16} M(0)+\epsilon, \quad \xi \geqslant x_{0}, t \in[0, T]$.

Lemma 10 Let the assumptions defined in Lemma 9 hold. Then there is $x_{0} \gg 1$ independent of $t$ such that

$$
\sup _{\xi \in\left[x_{0}, \infty\right)}|v(t, \xi)| \leqslant C_{17} M(0) \mathrm{e}^{-\mu_{2} t}
$$

for $t \in[0, T]$, where $C_{17}=C_{16}+1$. 
Hence we can obtain the following result.

Theorem 3 (a priori estimate) If assumptions in Theorem 2 hold and $v(t, \xi) \in X(-r, T)$ is a local solution of (4) with a given positive number $T$, then there are numbers $\delta_{1}>0,0<\mu<\min \left\{\mu_{1}, \mu_{2}\right\}$ and $C_{18}=\max \left\{C_{13},\left(C_{15}+C_{17}\right)^{2}\right\}$ independent of $T$ such that when $M(T) \leqslant \delta_{1}$,

$$
\begin{gathered}
\|v(t)\|_{C}^{2}+\|v(t)\|_{H_{\omega}^{2}}^{2} \\
\quad+\int_{0}^{t} \mathrm{e}^{-2 \mu(t-s)}\|v(s)\|_{H_{\omega}^{2}}^{2} \mathrm{~d} s \\
\leqslant C_{18} \mathrm{e}^{-2 \mu t}\left(\max _{s \in[-r, 0]}\left\|v_{0}(s)\right\|_{C}^{2}+\left\|v_{0}(0)\right\|_{H_{\omega}^{2}}^{2}\right) \\
+C_{18} \mathrm{e}^{-2 \mu t} \int_{-r}^{0}\left\|v_{0}(s)\right\|_{H_{\omega}^{2}}^{2} \mathrm{~d} s, t \in[0, T] .
\end{gathered}
$$

Proof: Combining the assumptions with (22), (23), and (26), we obtain (27) directly.

\section{STABILITY OF TRAVELLING WAVES}

This section is devoted to prove the stability of travelling wave solutions for (1). To obtain the exponential decay estimate, we give the following local existence result.

Proposition 1 (local existence) For the following problems with the initial value $\tau \geqslant 0$,

$$
\begin{gathered}
v_{t}(t, \xi)+c v_{\xi}(t, \xi)-d v_{\xi \xi}(t, \xi)-\partial_{1} F(\xi) v(t, \xi) \\
=\partial_{2} F(\xi) \int_{-\infty}^{\infty} J(y)[f(v+\phi)-f(\phi)] \mathrm{d} y+Q, \\
(t, \xi) \in(\tau, \infty) \times \mathbb{R}, \\
v(s, \xi)=u_{0}(s, \xi-c s)-\phi(\xi)=: v_{\tau}(s, \xi), \\
(s, \xi) \in[\tau-r, \tau] \times \mathbb{R} .
\end{gathered}
$$

If $\nu_{\tau}(s, \xi) \in X(\tau-r, \tau)$ and $M_{\tau}(0) \leqslant \delta_{2}$ for some constant $\delta_{2}>0$, then there is $t_{0}=t_{0}\left(\delta_{2}\right)>0$ such that $v(t, \xi) \in X\left(\tau-r, \tau+t_{0}\right)$ and $M_{\tau}\left(t_{0}\right) \leqslant$ $\sqrt{2(1+r)} M_{\tau}(0)$.

Next we give the following result for (4), which suggest Theorem 2 directly.

Theorem 4 (Stability) Suppose that $\left(A_{1}\right)-\left(A_{5}\right)$ hold. For a travelling wave solution $\phi(\xi)$ of (1) with $c>\tilde{c}$, $\tilde{c}$ is defined in Theorem 2, if $v_{0}(s, \xi) \in$ $C\left([-r, 0] ; C(\mathbb{R}) \cap H_{\omega}^{2}(\mathbb{R})\right) \cap L^{2}\left([-r, 0] ; H_{\omega}^{2}(\mathbb{R})\right)$ and $\lim _{\xi \rightarrow \infty} v_{0}(s, \xi)=: v_{0, \infty}(s) \in C[-r, 0]$ exists uniformly for $s \in[-r, 0]$, then there are numbers $\delta_{0}>0$ and $\mu>0$ independent of $v(t, \xi)$ such that when
$M_{\tau}(0) \leqslant \delta_{0}$, the unique solution $v(t, x)$ of (4) exists globally and satisfies

$$
\begin{array}{r}
\|v(t)\|_{C}^{2}+\|v(t)\|_{H_{\omega}^{2}}^{2}+\int_{0}^{t} \mathrm{e}^{-2 \mu(t-s)}\|v(s)\|_{H_{\omega}^{2}}^{2} \mathrm{~d} s \\
\leqslant C_{18} \mathrm{e}^{-2 \mu t}\left(\max _{s \in[-r, 0]}\left\|v_{0}(s)\right\|_{C}^{2}+\left\|v_{0}(0)\right\|_{H_{\omega}^{2}}^{2}\right) \\
+C_{18} \mathrm{e}^{-2 \mu t} \int_{-r}^{0}\left\|v_{0}(s)\right\|_{H_{\omega}^{2}}^{2} \mathrm{~d} s, t \in[0, \infty) .
\end{array}
$$

Proof: The proof is similar to the previous ones, thus we give the main points as follows. Since positive numbers $\delta_{1}, C_{18}$, and $\mu$ given in Theorem 3 are independent of $T$, by choosing $\delta_{0}=$ $\delta_{1} / \sqrt{2(1+r)}, \quad \delta_{2}=\max \left\{\sqrt{2 C_{18}(1+r)} M(0), \delta_{1}\right\}$, and $M(0) \leqslant \delta_{0}<\delta_{1} \leqslant \delta_{2}$, and by Proposition 1 , there is $t_{0}=t_{0}\left(\delta_{2}\right)>0$ such that $v(t, \xi) \in X\left(-r, t_{0}\right)$ and $M\left(t_{0}\right) \leqslant \sqrt{2(1+r)} M(0) \leqslant \delta_{1}$. Thus for $t \in$ $\left[0, t_{0}\right]$, Theorem 3 gives

$$
\begin{array}{r}
\|v(t)\|_{C}^{2}+\|v(t)\|_{H_{\omega}^{2}}^{2}+\int_{0}^{t} \mathrm{e}^{-2 \mu(t-s)}\|v(s)\|_{H_{\omega}^{2}}^{2} \mathrm{~d} s \\
\leqslant C_{18} \mathrm{e}^{-2 \mu t}\left(\max _{s \in[-r, 0]}\left\|v_{0}(s)\right\|_{C}^{2}+\left\|v_{0}(0)\right\|_{H_{\omega}^{2}}^{2}\right) \\
+C_{18} \mathrm{e}^{-2 \mu t} \int_{-r}^{0}\left\|v_{0}(s)\right\|_{H_{\omega}^{2}}^{2} \mathrm{~d} s, t \in\left[0, t_{0}\right] .
\end{array}
$$

When consider (28) at $\tau=t_{0}$, it clear that $M_{t_{0}}(0) \leqslant M\left(t_{0}\right) \leqslant \delta_{1} \leqslant \delta_{2}$, and by Theorem 3 we have $v(t, \xi) \in X\left(-r, 2 t_{0}\right)$ and $M_{t_{0}}\left(t_{0}\right) \leqslant$ $\sqrt{2(1+r)} M_{t_{0}}(0)$. Combining with

$$
\begin{aligned}
M_{t_{0}}(0) & =\sup _{\left[t_{0}-r, t_{0}\right]}\left(\|u(t)\|_{C(\mathbb{R})}^{2}+\|u(t)\|_{H_{\omega}^{2}(\mathbb{R})}^{2}\right) \\
& \leqslant \frac{\delta_{1}}{\sqrt{2(1+r)}},
\end{aligned}
$$

we obtain $M_{t_{0}}\left(t_{0}\right) \leqslant \delta_{1}$. Then

$$
\begin{aligned}
M\left(2 t_{0}\right) & =\sup _{\left[-r, 2 t_{0}\right]}\left(\|u(t)\|_{C(\mathbb{R})}^{2}+\|u(t)\|_{H_{\omega}^{2}(\mathbb{R})}^{2}\right) \\
& \leqslant \frac{\delta_{1}}{\sqrt{2(1+r)}} \leqslant \delta_{1} .
\end{aligned}
$$

Consequently, by Theorem 3, we can obtain the exponential decay for $t \in\left[0,2 t_{0}\right]$. By repeating this process, we can prove $v(t, \xi) \in X(-r, \infty)$ and (29) for $t \in[0, \infty)$.

\section{APPLICATIONS}

In this section we shall apply our consequences obtained in the former sections to some important models arising from practical problems. 


\section{Example 1}

Consider the case of (1) with the heat kernel $J(y)=\frac{1}{\sqrt{4 \pi \rho}} \mathrm{e}^{-y^{2} / 4 \rho}, \rho>0$. We obtain the following exponential stability of the travelling wave solutions for (1).

Corollary 1 Assume that $\left(A_{1}\right)-\left(A_{5}\right)$ hold. For the given travelling wave solution $\phi(\xi)$ of (1) with the speed $c>\hat{c}$, with $\hat{c}$ satisfying

$$
\frac{\hat{c} \lambda_{*}-d \lambda_{*}^{2}-\partial_{1} F(0,0)}{c_{*} \lambda_{*}-d \lambda_{*}^{2}-\partial_{1} F(0,0)}=\mathrm{e}^{\lambda_{*}^{2} \rho},
$$

if $u_{0}(s, x)-\phi(x+c s) \in C\left([-r, 0] ; C(\mathbb{R}) \cap H_{\omega}^{2}(\mathbb{R})\right) \cap$ $L^{2}\left([-r, 0] ; H_{\omega}^{2}(\mathbb{R})\right)$ and $\lim _{x \rightarrow \infty}\left[u_{0}(s, x)-\phi(x+\right.$ $c s)]=: v_{0, \infty} \in C[-r, 0]$ exists uniformly with $s \in$ $[-r, 0]$, then there are numbers $\delta_{0}>0$ and $\mu>0$ such that when

$$
\begin{aligned}
\max _{s \in[-r, 0]}\left\|\left(u_{0}-\phi\right)(s)\right\|_{C}^{2} & +\left\|\left(u_{0}-\phi\right)(0)\right\|_{H_{\omega}^{2}}^{2} \\
& +\int_{-r}^{0}\left\|\left(u_{0}-\phi\right)(s)\right\|_{H_{\omega}^{2}}^{2} \mathrm{~d} s \leqslant \delta_{0}^{2},
\end{aligned}
$$

the unique solution $u(t, x)$ of (1) and (2) exists globally and it satisfies

$$
\begin{array}{r}
u(t, x)-\phi(x+c t) \in C\left([-r, \infty) ; C(\mathbb{R}) \cap H_{\omega}^{2}(\mathbb{R})\right) \\
\cap L^{2}\left([-r, \infty) ; H_{\omega}^{2}(\mathbb{R})\right) \cap \mathscr{C}_{\text {unif }}[-r, \infty)
\end{array}
$$

and

$$
\sup _{x \in \mathbb{R}}|u(t, x)-\phi(x+c t)| \leqslant C \mathrm{e}^{-\mu t}, \quad t \geqslant 0 .
$$

Remark 2 The condition (31) implies that we may obtain the stability of the slower waves with wave speed near to the critical speed if $\rho$ is sufficiently small.

\section{Example 2}

Taking $F(x, y)=-\alpha_{1} x+\alpha_{2} y$ and $J(y)=\delta(y)$, a Dirac delta function, the equation (1) is reduced to

$$
\begin{aligned}
u_{t}(t, x)=d u_{x x}(t, x)-\alpha_{1} u(t, x) & \\
& +\alpha_{2} f(u(t-r, x)),
\end{aligned}
$$

where $\alpha_{1}, \alpha_{2}>0$. We assume the following two assumptions for (32):

$\left(H_{1}\right) f(K)=\alpha_{1} K / \alpha_{2}, f(x)>\alpha_{1} x / \alpha_{2}, x \in(0, K]$.

$\left(H_{2}\right) f^{\prime}(K) \leqslant \alpha_{1} / \alpha_{2}$ with any $r>0$, or $f^{\prime}(K)>$ $\alpha_{1} / \alpha_{2}$ with $0<r<\tilde{r}$, where

$$
\tilde{r}:=\frac{\pi-\arctan \left[\alpha_{1}^{-1} \sqrt{\left(\alpha_{2} f^{\prime}(K)\right)^{2}-\alpha_{1}^{2}}\right]}{\sqrt{\left(\alpha_{2} f^{\prime}(K)\right)^{2}-\alpha_{1}^{2}}} .
$$

Set $\mathscr{P}(\lambda, c)=d \lambda^{2}-c \lambda-\alpha_{1}+\alpha_{2} f^{\prime}(0) \mathrm{e}^{-\lambda c r}$. Then there exist $c_{*}$ and $\lambda_{*}$ such that $\mathscr{P}\left(\lambda_{*}, c_{*}\right)=0$. From Ref. 4, (32) admits a travelling wave solution $\phi(\xi)$, $\xi=x+c t$, for any $c>c_{*}$ such that

$$
\begin{aligned}
& 0<\phi(\xi) \leqslant K, \quad \phi(-\infty)=0, \\
& 0<\liminf _{\xi \rightarrow \infty} \phi(\xi) \leqslant \limsup _{\xi \rightarrow \infty} \phi(\xi) \leqslant K .
\end{aligned}
$$

Clearly, $\partial_{1} F(x, y)=-\alpha_{1}<0, \partial_{2} F(x, y)=\alpha_{2}>0$, then $\lim _{\xi \rightarrow \infty} \phi(\xi)=K$. We obtain the following stability result of (32).

Corollary 2 Assume that $\left(A_{2}\right),\left(H_{1}\right)-\left(H_{2}\right)$ hold. For a travelling wave solution $\phi(\xi)$ of (32) with the speed $c>c_{*}$, if $u_{0}(s, x)-\phi(x+c s) \in C([-r, 0] ; C(\mathbb{R}) \cap$ $\left.H_{\omega}^{2}(\mathbb{R})\right) \cap L^{2}\left([-r, 0] ; H_{\omega}^{2}(\mathbb{R})\right)$ and $\lim _{x \rightarrow \infty}\left[u_{0}(s, x)-\right.$ $\phi(x+c s)]=: v_{0, \infty} \in C[-r, 0]$ exists uniformly with $s \in[-r, 0]$, then there are $\delta_{0}>0$ and $\mu>0$ such that when

$$
\begin{aligned}
\max _{s \in[-r, 0]}\left\|\left(u_{0}-\phi\right)(s)\right\|_{C}^{2} & +\left\|\left(u_{0}-\phi\right)(0)\right\|_{H_{\omega}^{2}}^{2} \\
& +\int_{-r}^{0}\left\|\left(u_{0}-\phi\right)(s)\right\|_{H_{\omega}^{2}}^{2} \mathrm{~d} s \leqslant \delta_{0}^{2},
\end{aligned}
$$

the unique solution $u(t, x)$ of (32) and (2) exists globally and satisfies

$u(t, x)-\phi(x+c t) \in C\left([-r, \infty) ; C(\mathbb{R}) \cap H_{\omega}^{2}(\mathbb{R})\right)$

$$
\cap L^{2}\left([-r, \infty) ; H_{\omega}^{2}(\mathbb{R})\right) \cap \mathscr{C}_{\text {unif }}[-r, \infty)
$$

and

$$
\sup _{x \in \mathbb{R}}|u(t, x)-\phi(x+c t)| \leqslant C \mathrm{e}^{-\mu t}, \quad t \geqslant 0 .
$$

\section{Example 3}

Consider the following general population model, which derives from the evolution of the mature population with an age structure of a single species ${ }^{1}$,

$$
\begin{aligned}
& u_{t}(t, x)=d \Delta u(t, x)-g(u(t, x)) \\
& +h(u(t, x)) \int_{-\infty}^{\infty} J(x-y) f(u(t-r, y)) \mathrm{d} y,
\end{aligned}
$$

where $d>0, r \geqslant 0$. We assume the following assumptions.

$\left(P_{1}\right) h(x), g(x) \in C^{2}([0, K] \times[0, f(K)], \mathbb{R}), g(0)=$ $0, h(K) f(K)=g(K), h(x) f(x)>g(x), h(x)>$ $0, h^{\prime}(x) \leqslant 0, g^{\prime}(x) \geqslant g^{\prime}(0) \geqslant 0$ for $x \in(0, K], K$ is defined in $A_{2}$.

$\left(P_{2}\right)$ There exists $0<\theta<K$ such that $f$ is increasing on $[0, \theta]$, and $0<\eta<K$ such that for any $y \in$ $(0, \eta), h(x) y=g(x)$ has a solution $x \in(0, \theta)$. 
$\left(P_{3}\right) h(K) f^{\prime}(K)+h^{\prime}(K) f(K)-g^{\prime}(K) \leqslant 0$ with any time-delay $r>0$, or $h(K) f^{\prime}(K)+h^{\prime}(K) f(K)-$ $g^{\prime}(K)>0$ with time-delay $0<r<\tilde{r}$, when

$$
\tilde{r}:=\frac{\pi-\arctan \left(\left(h^{\prime}(K) f(K)-g^{\prime}(K)\right)^{-1} \tilde{M}\right)}{\tilde{M}},
$$

$$
\tilde{M}=\sqrt{\left(h(K) f^{\prime}(K)\right)^{2}-\left(h^{\prime}(K) f(K)-g^{\prime}(K)\right)^{2}} .
$$

Taking $F(x, y)=-g(x)+h(x) y$ in (1) to obtain (33). It is easy to check that $u \equiv 0$ and $u \equiv K$ are two constant equilibria of (33). Clearly,

$$
\begin{aligned}
& \partial_{1} F(x, y)=-g^{\prime}(x)+h^{\prime}(x) y \leqslant \partial_{1} F(0,0)<0, \\
& 0<\partial_{2} F(x, y)=h(x) \leqslant \partial_{2} F(0,0), \\
& F(x, y) \leqslant-g^{\prime}(0) x+h(0) y, \\
& \text { for }(x, y) \in[0, K] \times[0, f(K)] . \text { Set } \\
& \mathscr{P}(\lambda, c)=d \lambda^{2}-c \lambda-g \\
&+h^{\prime}(0) f^{\prime}(0) \int_{-\infty}^{\infty} J(y) \mathrm{e}^{-\lambda(y+c r)} \mathrm{d} y .
\end{aligned}
$$

Thus there exist $c_{*}$ and $\lambda_{*}$ such that $\mathscr{P}\left(\lambda_{*}, c_{*}\right)=0$. From Ref. 1, (33) admits a travelling wave solution $\phi(\xi), \xi=x+c t$, for any $c>c_{*}$ such that

$$
0<\phi(\xi) \leqslant K, \phi(-\infty)=0, \lim _{\xi \rightarrow \infty} \phi(\xi)=K,
$$

and obtain the following stability result of (33).

Corollary 3 Assume that $\left(A_{1}\right)-\left(A_{2}\right)$ and $\left(P_{1}\right)-\left(P_{3}\right)$ hold. For a travelling wave solution $\phi(\xi)$ of (33) with the speed $c>\bar{c}$, with $\bar{c}$ satisfying

$$
\begin{aligned}
\bar{c} \lambda_{*}-h^{\prime}(0) f^{\prime}(0)\left(\int_{-\infty}^{\infty} J(y) \mathrm{e}^{-2 \lambda_{*}(y+\bar{c} r)} \mathrm{d} y\right)^{1 / 2} & \\
& =2 d \lambda_{*}^{2}-g^{\prime}(0),
\end{aligned}
$$

if $u_{0}(s, x)-\phi(x+c s) \in C\left([-r, 0] ; C(\mathbb{R}) \cap H_{\omega}^{2}(\mathbb{R})\right) \cap$ $L^{2}\left([-r, 0] ; H_{\omega}^{2}(\mathbb{R})\right)$ and $\lim _{x \rightarrow \infty}\left[u_{0}(s, x)-\phi(x+\right.$ $c s)]=: v_{0, \infty} \in C[-r, 0]$ exists uniformly with $s \in$ $[-r, 0]$, then there are $\delta_{0}>0$ and $\mu>0$ such that when

$$
\begin{aligned}
\max _{s \in[-r, 0]}\left\|\left(u_{0}-\phi\right)(s)\right\|_{C}^{2} & +\left\|\left(u_{0}-\phi\right)(0)\right\|_{H_{\omega}^{2}}^{2} \\
& +\int_{-r}^{0}\left\|\left(u_{0}-\phi\right)(s)\right\|_{H_{\omega}^{2}}^{2} \mathrm{~d} s \leqslant \delta_{0}^{2},
\end{aligned}
$$

the unique solution $u(t, x)$ of (33) and (2) exists globally and satisfies

$$
\begin{aligned}
& u(t, x)-\phi(x+c t) \in C\left([-r, \infty) ; C(\mathbb{R}) \cap H_{\omega}^{2}(\mathbb{R})\right) \\
& \cap L^{2}\left([-r, \infty) ; H_{\omega}^{2}(\mathbb{R})\right) \cap \mathscr{C}_{\text {unif }}[-r, \infty)
\end{aligned}
$$

and

$$
\sup _{x \in \mathbb{R}}|u(t, x)-\phi(x+c t)| \leqslant C \mathrm{e}^{-\mu t}, \quad t \geqslant 0 .
$$

Acknowledgements: This work was supported by the National Natural Science Foundation of China (No. 11471197) and the Natural Science Foundation of Shanxi Province (No. 201601D202002).

\section{REFERENCES}

1. Ma S (2007) Traveling waves for nonlocal delayed diffusion equations via auxiliary equation. $J$ Differ Equations 237, 259-77.

2. Duehring D, Huang WZ (2007) Periodic traveling waves for diffusion equations with time delayed and non-local responding reaction. J Dyn Differ Equ 19, $457-77$.

3. Faria T, Trofimchuk S (2006) Nonmonotone traveling waves in a single species reaction-diffusion equation with delay. $J$ Differ Equations 228, 357-76.

4. Wang HY (2009) On the existence of traveling waves for delayed reaction-diffusion equations. $J$ Differ Equations 247, 887-905.

5. Xu ZQ, Xiao D (2016) Spreading speeds and uniqueness of traveling waves for a reaction diffusion equation with spatio-temporal delays. $J$ Differ Equations 260, 268-303.

6. Schaaf KW (1987) Asymptotic behavior and traveling wave solutions for parabolic functional-differential equations. Trans Amer Math Soc 302, 587-615.

7. Mei M, So JWH, Li MY, Shen SSP (2004) Asymptotic stability of traveling waves for the Nicholson's blowflies equation with diffusion. Proc Roy Soc Edinburgh Sect A 134, 579-94.

8. Lv GY, Wang MX (2010) Nonlinear stability of traveling wave fronts for delayed reaction diffusion equations. Nonlinearity 23, 1609-30.

9. Wu SL, Li WT, Liu SY (2009) Asymptotic stability of traveling wave fronts in nonlocal reaction-diffusion equations with delay. $J$ Math Anal Appl 360, 439-58.

10. Lin CK, Mei M (2010) On traveling wavefronts of Nicholson's blowflies equations with diffusion. Proc Roy Soc Edinburgh Sect A 140, 135-52.

11. Mei M (2009) Stability of traveling wavefronts for time-delay reaction-diffusion equations. Discrete Contin Dyn Syst, Suppl, 526-35.

12. Wu SL, Zhao HQ, Liu SY (2011) Asymptotic stability of traveling waves for delayed reaction-diffusion equations with crossing-monostability. $Z$ Angew Math Phys 62, 377-97.

13. Lin CK, Lin CT, Lin YP, Mei M (2014) Exponential stability of nonmonotone traveling waves for Nicholson's blowflies equation. SIAM J Math Anal 46, 1053-84. 